\title{
Performance of Some Tomato (Solanum lycopersicum L.) Genotypes in Summer and Winter Seasons
}

\author{
M. M. Alam Patwary ${ }^{1}$, M. Mizanur Rahman², Shahabuddin Ahmad ${ }^{3}$, M. A. Khaleque \\ Miah $^{2}$ and M. H. Rahman ${ }^{1}$ \\ ${ }^{1}$ Agricultural Research Station, Bangladesh Agricultural Research Institute, Pahartali, Chittagong; \\ ${ }^{2}$ Bangabandhu Sheikh Mujibur Rahman Agricultural University, Gazipur, Bangladesh; ${ }^{3}$ Horticulture \\ Research Centre, Bangladesh Agricultural Research Institute, Gazipur, Bangladesh
}

*Corresponding author and Email: kbdmahbub69@gmail.com

Received: 10 October 2013

Accepted: 13 December 2014

\begin{abstract}
An experiment was conducted at the Vegetable Research Field of Olericulture Division, Horticulture Research Centre, Bangladesh Agricultural Research Institute, Gazipur during October 2006 to March 2007 and May to September 2007 to evaluate the performance of tomato (Solanum lycopersicum) genotypes in summer and winter seasons. Early flowering was observed in summer compared to winter. Pollen viability decreased greatly during summer ranging from $30.44 \%$ in TMS 003 to 86.08 $\%$ in C 11 compared to that in winter $(70.33 \%$ in C 61 to $100.00 \%$ in VRT 002). Fruit set (\%) markedly decreased in summer, which ranged from $4.69 \%$ in TMS 017 to $39.15 \%$ in C 51 while it ranged from and $49.00 \%$ in TMS 008 to $90.01 \%$ in HT 017 during winter. During summer, fruit set $(\%)$ exhibited positive significant and correlation with viable pollen grains $(\%)$. Yield per plant ranged from $1224 \mathrm{~g}$ in C 61 to $2670 \mathrm{~g}$ in VRT 003 and $37 \mathrm{~g}$ in TMS 015 to $94 \mathrm{~g}$ in C 11 in winter. The genotypes HT 019, C 11, C 21, C 41, C 51, HT 016 and HT 017 exhibited a considerable heat tolerance in relation to fruit setting ability.
\end{abstract}

Keywords: Tomato, heat tolerance, pollen viability

\section{Introduction}

Production of tomato (Solanum lycopersicum L.) in Bangladesh is huge in winter mainly from December to February and less in early and late winter months, but not in summer. Thus, the production of tomato in Bangladesh is confined only in the winter season. There are many varieties, such as Ratan and Roma VF are exclusively grown in winter. All these varieties are heat sensitive and usually fail to set fruit under high temperature. Because of high price and demand of tomato in summer, the need for suitable variety summer has long been demanded. The optimum day/night temperatures for fruit set in tomato is in the range of 26$32^{\circ} \mathrm{C} / 15-20^{\circ} \mathrm{C}$ (Kuo et al., 1979). However, there is usually genotypic variation in their fruit setting ability at high temperature (Charles and Harris, 1972). Because of favourable growing conditions and high demand, we have many varieties for winter and introduced from abroad. But, limited efforts have been given so far to overcome the high temperature barrier preventing fruit set in summer and rainy seasons. Moreover, demand has been created among farmers for summer variety. 
BARI has been developed some heat tolerant open pollinated $(\mathrm{OP})$ and $\mathrm{F}_{1}$ Hybrid tomato varieties such as BARI Tomato-4, BARI Tomato-5, BARI Tomato-10 (Anupama), BARI Tomato-13, BARI Hybrid Tomato-3 and BARI Hybrid Tomato-4 ( BARI, 2011). But these varieties are not enough to meet up the existing demand. However, limited research was conducted to improve yield and quality of tomato during summer in Bangladesh. To develop cultivars for hot summer, selection of genotypes which are capable of setting fruits under heat stress is needed.

There are many factors responsible for fruit setting process which are needed to be explored under high temperature stress. Comparative performances of the genotypes under normal and high temperature conditions are also needed for better understanding of the reaction of individual component factor of pollen viability, fruit setting and yield attributing parameters aiming to select suitable heat tolerant genotypes. Therefore, considering the present need, the study was under taken to compare genotypic differences in respect of different yield contributing characters during winter and summer seasons for selecting superior heat tolerant genotypes of tomatoes suitable for summer season.

\section{Materials and Methods}

The experiment was conducted at the Vegetable Research Field of Olericulture Division, Horticulture Research Centre (HRC), Bangladesh Agricultural Research Institute (BARI), Gazipur during October 2006 to March 2007 and May to September 2007. The location of the experiment was at $24{ }^{0} \mathrm{~N}$ latitude, $92.25{ }^{0} \mathrm{E}$ longitude and 8.4 metre elevation from the sea level (Anon., 1995). It was situated in the subtropical climatic zone, characterized by heavy rainfall during the month of May to August and scanty rainfall during the rest of the year. Data on temperature and relative humidity during the study period were recorded and were presented in Table 5. Seeds of twenty heat tolerant tomato genotypes as selected on the basis of literatures were collected from Olericulture Division, HRC, Bangladesh Agricultural Research Institute were used as plant materials.

Seeds were sown and seedlings were grown both in winter and summer seasons. For winter and summer seasons seeds were sown on 27 October 2006 and 08 May, 2007, respectively. The seedlings were transplanted in the main experimental field at 35 days after sowing during winter and at 25 days during summer. Plants were given support by bamboo sticks in both seasons.

Winter crops were grown in open field and the summer crops were grown under transparent polytunnel. The polytunnels were 2.3 meter wide. Each polytunnel contained two 1.0 meter wide bed, with $30 \mathrm{~cm}$ drain in-between. The experiment was laid out in randomized complete block design (RCBD) with three replications both in summer and winter. The unit plot size was $3.2 \mathrm{~m} \mathrm{x} 1.0 \mathrm{~m}$ and the plants were spaced 40x60 $\mathrm{cm}$ on beds. The layout for winter crop was also same but without polytunnel. Chemical fertilizers@550 kg urea, 450 kg TSP, 250 kg MoP, $120 \mathrm{~kg}$ Zypsum, $2 \mathrm{~kg}$ Boron and 10 metric ton cow dung per hectare were applied. Half of the cow dung and the entire quantity of TSP were applied during final land preparation. The pits were prepared one week before transplanting seedlings. The remaining cow dung and $1 / 3$ of $\mathrm{MoP}$ were applied in pits during prepared. Topdressing was done in 3 equal installments at 10 , 25 and 40 days after transplanting to apply the entire urea and rest $2 / 3$ of MoP. Weeding and mulching were done followed by top-dressing and irrigation at 15 days interval.

Data on days to $50 \%$ flowering, flowers per cluster, viable pollen, fruit set $(\%)$, fruits per plant (number), TSS content, fruit weight (g), fruit yield per plant $(\mathrm{g})$, fruit length $(\mathrm{cm})$, fruit diameter $(\mathrm{cm})$, branches per plant, plant height $(\mathrm{cm})$, seeds per fruit and 1000 seeds weight were recorded. The recorded data on different characters were analyzed statistically using MSTAT-C programme where means were 
compared by DMRT to find out the variation among the different genotypes.

\section{Results and Discussion}

\subsection{Days to $50 \%$ flowering}

Significant variation was observed among the genotypes in respect of days to $50 \%$ flowering in both seasons. More days were required for flowering by all the genotypes during summer season compared to that in winter, indicating delayed flower formation under summer seasons condition (Table 1). In summer, 43.33 and 71.3 days were required for $50 \%$ flowering, respectively for C 51 and VRT 001 which reduced to 38.33 and 55.00 days in winter. Kuo et al., (1979) also reported that flower formation is influenced by high temperature stress; which is consistent to the present study.

\subsection{Flowers per cluster}

Marked reduction in flower number per cluster were observed in all the genotypes in summer compared to winter except C 11, VRT, TMS 005, TMS 008, TMS o13 and TMS o17, which varied from 4.18 to 9.92 in summer and 5.09 to 11.09 in winter (Table 1). Charles and Harris (1972) reported decrease of flower production with increased temperature.
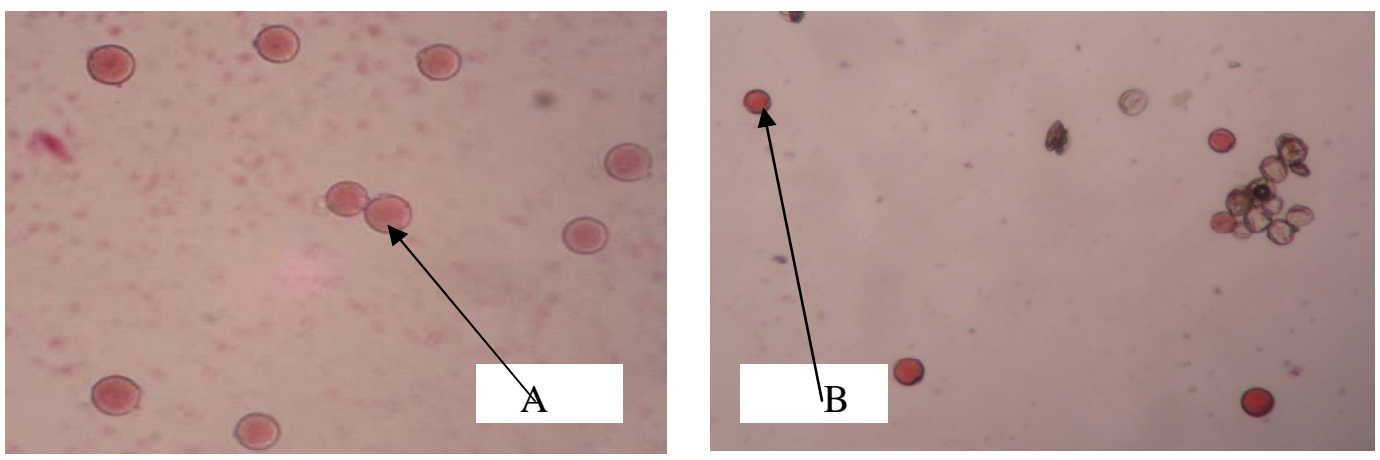

Figure. 1. Pollen grains: A. More viable pollens during winter; B. Less viable pollens during summer 
Table 1. Yield contributing parameters of tomato genotypes during winter and summer seasons

\begin{tabular}{|c|c|c|c|c|c|c|c|c|c|}
\hline \multirow{2}{*}{\multicolumn{2}{|c|}{ Genotypes }} & \multicolumn{2}{|c|}{ Days to $50 \%$ flowering } & \multicolumn{2}{|c|}{ No. of flowers/cluster } & \multicolumn{2}{|c|}{ No. of viable pollens (\%) } & \multicolumn{2}{|c|}{ No. of fruit set (\%) } \\
\hline & & Winter & Summer & Winter & Summer & Winter & Summer & Winter & Summer \\
\hline C 11 & & 44.7 e-h & $56.3 \mathrm{~d}$ & 7.74 e-h & $8.11 \mathrm{~b}-\mathrm{d}$ & $89.66 \mathrm{a}-\mathrm{d}$ & $86.08 \mathrm{a}$ & $81.33 \mathrm{a}-\mathrm{d}$ & $29.46 \mathrm{~b}$ \\
\hline C 21 & & $42.3 \mathrm{~g}-\mathrm{i}$ & $48.3 \mathrm{fg}$ & $8.62 \mathrm{c}-\mathrm{d}$ & $7.40 \mathrm{~b}-\mathrm{g}$ & $92.67 \mathrm{a}-\mathrm{c}$ & $74.40 \mathrm{ab}$ & $83.33 \mathrm{a}-\mathrm{c}$ & $37.47 \mathrm{a}$ \\
\hline C 41 & & $41.3 \mathrm{hi}$ & $52.7 \mathrm{~d}-\mathrm{f}$ & $8.95 \mathrm{~cd}$ & $7.61 \mathrm{~b}-\mathrm{f}$ & $95.00 \mathrm{a}-\mathrm{c}$ & $77.40 \mathrm{ab}$ & $75.67 \mathrm{~b}-\mathrm{e}$ & $27.05 \mathrm{bc}$ \\
\hline C 51 & & $38.3 \mathrm{i}$ & $43.3 \mathrm{~h}$ & $6.03 \mathrm{jk}$ & $5.41 \mathrm{~h}-\mathrm{k}$ & $98.00 \mathrm{ab}$ & $68.20 \mathrm{bc}$ & $81.67 \mathrm{a}-\mathrm{d}$ & $39.15 \mathrm{a}$ \\
\hline C 61 & & $42.3 \mathrm{~g}-\mathrm{i}$ & $55.3 \mathrm{~d}-\mathrm{e}$ & $7.87 \mathrm{e}-\mathrm{h}$ & $6.01 \mathrm{f}-\mathrm{j}$ & $70.33 \mathrm{e}$ & $53.89 \mathrm{~d}-\mathrm{f}$ & $72.00 \mathrm{~d}-\mathrm{f}$ & $21.33 \mathrm{~cd}$ \\
\hline C 71 & & $43.7 \mathrm{f}-\mathrm{i}$ & 50.7 ef & $9.43 \mathrm{bc}$ & $7.97 \mathrm{~b}-\mathrm{e}$ & $90.33 \mathrm{a}-\mathrm{d}$ & $46.09 \mathrm{f}$ & $74.67 \mathrm{~b}-\mathrm{e}$ & $20.63 \mathrm{~cd}$ \\
\hline HT 016 & & 44.7 e-h & $52.0 \mathrm{~d}-\mathrm{f}$ & $11.09 \mathrm{a}$ & $9.92 \mathrm{a}$ & $97.33 \mathrm{a}-\mathrm{c}$ & $68.40 \mathrm{bc}$ & $84.67 \mathrm{ab}$ & $25.30 \mathrm{bc}$ \\
\hline HТ 017 & & $43.7 \mathrm{f}-\mathrm{i}$ & $48.0 \mathrm{fg}$ & $9.30 \mathrm{bc}$ & $8.75 \mathrm{ab}$ & $88.00 \mathrm{~b}-\mathrm{d}$ & $76.40 \mathrm{ab}$ & $90.01 \mathrm{a}$ & $24.66 \mathrm{bc}$ \\
\hline НТ 019 & & $39.0 \mathrm{i}$ & $46.0 \mathrm{gh}$ & $10.06 \mathrm{~b}$ & $8.34 \mathrm{a}-\mathrm{c}$ & $99.00 \mathrm{ab}$ & $82.47 \mathrm{a}$ & $81.33 \mathrm{a}-\mathrm{d}$ & $29.27 \mathrm{~b}$ \\
\hline VRT 001 & & $55.0 \mathrm{a}$ & $71.3 \mathrm{a}$ & $6.08 \mathrm{jk}$ & $4.55 \mathrm{jk}$ & $94.66 \mathrm{a}-\mathrm{c}$ & $51.40 \mathrm{~d}-\mathrm{e}$ & $77.00 \mathrm{~b}-\mathrm{e}$ & $12.63 \mathrm{e}-\mathrm{g}$ \\
\hline VRT 002 & & 49.7 a-e & $67.3 \mathrm{a}-\mathrm{c}$ & 7.78 e-h & $8.52 \mathrm{a}-\mathrm{c}$ & $100.00 \mathrm{a}$ & $56.00 \mathrm{c}-\mathrm{f}$ & $70.00 \mathrm{ef}$ & $9.17 \mathrm{f}-\mathrm{h}$ \\
\hline VRT 003 & & 49.7 a-e & $66.0 \mathrm{bc}$ & $8.17 \mathrm{~d}-\mathrm{g}$ & $5.71 \mathrm{~g}-\mathrm{k}$ & $94.67 \mathrm{a}-\mathrm{c}$ & $59.25 \mathrm{c}-\mathrm{f}$ & $74.33 \mathrm{~b}-\mathrm{e}$ & $13.58 \mathrm{ef}$ \\
\hline TMS 003 & & $46.3 \mathrm{~d}-\mathrm{h}$ & $68.0 \mathrm{ab}$ & $5.67 \mathrm{k}-1$ & $5.20 \mathrm{i}-\mathrm{k}$ & $94.33 \mathrm{a}-\mathrm{c}$ & $30.44 \mathrm{~g}$ & $78.00 \mathrm{~b}-\mathrm{e}$ & $6.01 \mathrm{~h}$ \\
\hline TMS 005 & & $47.7 \mathrm{c}-\mathrm{g}$ & $53.3 \mathrm{de}$ & 7.14 h-i & $8.37 \mathrm{a}-\mathrm{c}$ & $86.33 \mathrm{~cd}$ & 50.55 ef & $56.00 \mathrm{gh}$ & $5.48 \mathrm{~h}$ \\
\hline TMS 008 & & $54.3 \mathrm{ab}$ & $68.3 \mathrm{ab}$ & 5.091 & $7.01 \mathrm{c}-\mathrm{h}$ & $94.67 \mathrm{a}-\mathrm{c}$ & $60.80 c-e$ & $49.00 \mathrm{~h}$ & $13.89 \mathrm{ef}$ \\
\hline TMS 011 & & $52.3 \mathrm{a}-\mathrm{c}$ & $68.3 \mathrm{ab}$ & $7.30 \mathrm{~g}-\mathrm{i}$ & $6.52 \mathrm{~d}-\mathrm{i}$ & $97.33 \mathrm{a}-\mathrm{c}$ & $58.16 \mathrm{c}-\mathrm{f}$ & $63.67 \mathrm{fg}$ & $5.47 \mathrm{~h}$ \\
\hline TMS 013 & & 49.7 a-e & $55.0 \mathrm{de}$ & $7.43 \mathrm{f}-\mathrm{i}$ & 7.59 b-f & $95.00 \mathrm{a}-\mathrm{c}$ & $48.12 \mathrm{ef}$ & $59.00 \mathrm{~g}$ & $5.99 \mathrm{gh}$ \\
\hline TMS 015 & & $47.0 \mathrm{c}-\mathrm{g}$ & $67.7 \mathrm{ab}$ & $6.59 \mathrm{ij}$ & $4.18 \mathrm{k}$ & $81.33 \mathrm{~d}$ & $46.30 \mathrm{f}$ & $78.00 \mathrm{~b}-\mathrm{e}$ & $10.67 \mathrm{f}-\mathrm{h}$ \\
\hline TMS 017 & & $48.0 \mathrm{c}-\mathrm{f}$ & $69.0 \mathrm{ab}$ & $5.59 \mathrm{kl}$ & $6.98 \mathrm{c}-\mathrm{h}$ & $92.00 \mathrm{a}-\mathrm{c}$ & $51.10 \mathrm{~d}-\mathrm{f}$ & $78.00 \mathrm{~b}-\mathrm{e}$ & $4.69 \mathrm{~h}$ \\
\hline BARI 6 & & $50.7 \mathrm{a}-\mathrm{d}$ & $63.0 \mathrm{c}$ & $8.37 \mathrm{~d}-\mathrm{f}$ & $6.29 \mathrm{e}-\mathrm{i}$ & $93.33 \mathrm{a}-\mathrm{c}$ & $64.91 b-d$ & $74.00 \mathrm{c}-\mathrm{e}$ & $17.87 \mathrm{de}$ \\
\hline $\mathrm{CV}(\%)$ & & 6.08 & 4.37 & 6.63 & 12.78 & 6.17 & 12.20 & 10.19 & 7.19 \\
\hline $\begin{array}{l}\text { Level } \\
\text { significance }\end{array}$ & of & $* *$ & $* *$ & $* *$ & $* *$ & $* *$ & $* *$ & $* *$ & $* *$ \\
\hline
\end{tabular}

Means in a column followed by the same letters are not significantly different at $1 \%$ level of probability by DMRT 
The genotypes C 51, C 21, C 11, HT 019 and C 41 had high number of fruit set during summer (39.15, 37.47, 29.46, 29.27 and $27.05 \%$, respectively) among the genotypes (Table 1). In winter, the highest fruit set $(90.01 \%)$ was observed in the genotype HT 017 closely followed by HT 016 (84.67 \%), C 21 (83.33\%), and C $51(81.67 \%)$, while the lowest $(49.00 \%)$ was observed in the genotype TMS 008. These findings in agreement with the results reported by Hanna and Hernandez (1979), who reported that fruit set in a particular genotype viz., L 401 dropped from $78.1 \%$ in spring to as low as 1.2 $\%$ in summer.

In these study $50 \%$ genotypes viz., C 51, C 21, C 11, HT 019, C 41, HT 016, HT 017, C 61, C
71 and BARI 6 provided more than $17 \%$ fruit set. Remaining $50 \%$ genotypes produced less than $14 \%$ fruit as they flowered. Out of them, only 4 lines such as TMS 008, VRT 003, TMS 003 and TMS 005 ranged from $13.89 \%$ to 10.67 $\%$ and the rest genotypes set fruit ranging from $4.69 \%$ to $9.17 \%$, in these genotypes. Ahmad (2002) also observed varying level of fruit set in both the seasons.

\subsection{Fruits per plant}

The genotypes differed significantly with respect to number of fruits per plant in both the seasons. In winter, the number of fruit per plant was higher (16.9-89.3 per plant) and it was only 1.1330.2 per plant in summer (Table 2 ), whereas in winter it was 16.93 to 89.33 .

Table 2. Performances of tomato genotypes during winter and summer seasons for fruits per plant and TSS

\begin{tabular}{|c|c|c|c|c|}
\hline \multirow[t]{2}{*}{ Genotype } & \multicolumn{2}{|c|}{ Number of fruits / plant } & \multicolumn{2}{|l|}{ TSS (\%) } \\
\hline & Winter & Summer & Winter & Summer \\
\hline C 11 & $39.33 \mathrm{~cd}$ & $23.42 \mathrm{~b}$ & $4.89 \mathrm{de}$ & $4.43 \mathrm{a}-\mathrm{c}$ \\
\hline C 21 & $46.93 \mathrm{c}$ & $21.13 b c$ & $4.98 \mathrm{c}-\mathrm{e}$ & $4.28 \mathrm{~b}-\mathrm{d}$ \\
\hline C 41 & $47.20 \mathrm{c}$ & $18.20 \mathrm{~cd}$ & $4.81 \mathrm{e}$ & $4.23 \mathrm{~b}-\mathrm{e}$ \\
\hline C 51 & $36.00 \mathrm{de}$ & $19.13 \mathrm{~cd}$ & $5.28 \mathrm{bc}$ & $4.08 \mathrm{c}-\mathrm{f}$ \\
\hline C 61 & $35.13 \mathrm{~d}-\mathrm{f}$ & $15.33 \mathrm{~d}$ & $5.08 \mathrm{c}-\mathrm{e}$ & $3.76 \mathrm{fg}$ \\
\hline C 71 & $32.73 \mathrm{~d}-\mathrm{g}$ & $10.95 \mathrm{e}$ & $4.93 \mathrm{c}-\mathrm{e}$ & $4.37 \mathrm{bc}$ \\
\hline HТ 016 & $81.67 \mathrm{~b}$ & $30.20 \mathrm{a}$ & $4.40 \mathrm{f}$ & $4.23 \mathrm{~b}-\mathrm{e}$ \\
\hline HТ 017 & $89.33 \mathrm{a}$ & $24.80 \mathrm{~b}$ & $4.97 \mathrm{c}-\mathrm{e}$ & $4.61 \mathrm{ab}$ \\
\hline НТ 019 & $43.72 \mathrm{c}$ & $21.77 \mathrm{bc}$ & $5.01 \mathrm{c}-\mathrm{e}$ & $4.53 \mathrm{ab}$ \\
\hline VRT 001 & $26.27 \mathrm{~g}-\mathrm{i}$ & $1.53 \mathrm{f}$ & $4.95 \mathrm{c}-\mathrm{e}$ & $3.89 \mathrm{~d}-\mathrm{g}$ \\
\hline VRT 002 & $28.67 \mathrm{e}-\mathrm{i}$ & $2.80 \mathrm{f}$ & $4.83 \mathrm{e}$ & $4.11 \mathrm{c}-\mathrm{f}$ \\
\hline VRT 003 & 30.67 e-h & $2.60 \mathrm{f}$ & $5.21 \mathrm{~b}-\mathrm{d}$ & $4.24 \mathrm{~b}-\mathrm{e}$ \\
\hline TMS 003 & $28.80 \mathrm{e}-\mathrm{i}$ & $1.40 \mathrm{f}$ & $5.07 \mathrm{c}-\mathrm{e}$ & $3.93 \mathrm{~d}-\mathrm{g}$ \\
\hline TMS 005 & $26.87 \mathrm{f}-\mathrm{i}$ & $1.67 \mathrm{f}$ & $5.66 \mathrm{a}$ & $3.87 \mathrm{e}-\mathrm{g}$ \\
\hline TMS 008 & $16.93 \mathrm{j}$ & $3.03 \mathrm{f}$ & $4.37 \mathrm{f}$ & $3.97 \mathrm{~d}-\mathrm{g}$ \\
\hline TMS 011 & $21.93 \mathrm{ij}$ & $1.87 \mathrm{f}$ & $5.03 \mathrm{c}-\mathrm{e}$ & $3.89 \mathrm{~d}-\mathrm{g}$ \\
\hline TMS 013 & $24.00 \mathrm{~h}-\mathrm{j}$ & $2.00 \mathrm{f}$ & $5.47 \mathrm{ab}$ & $3.39 \mathrm{~h}$ \\
\hline TMS 015 & $30.73 \mathrm{e}-\mathrm{h}$ & $1.13 \mathrm{f}$ & $5.13 \mathrm{c}-\mathrm{e}$ & $3.72 \mathrm{f}-\mathrm{h}$ \\
\hline TMS 017 & $25.20 \mathrm{~g}-\mathrm{i}$ & $1.93 \mathrm{f}$ & $5.67 \mathrm{a}$ & $3.63 \mathrm{gh}$ \\
\hline BARI 6 & $25.07 \mathrm{~g}-\mathrm{i}$ & $5.47 \mathrm{f}$ & $5.52 \mathrm{ab}$ & $4.77 \mathrm{a}$ \\
\hline $\mathrm{CV}(\%)$ & 12.01 & 22.11 & 3.66 & 5.02 \\
\hline Level of significance & $* *$ & $* *$ & $* *$ & $* *$ \\
\hline
\end{tabular}

Means in a column followed by the same letters are not significantly different at $1 \%$ level of probability by DMRT 
The maximum fruits were recorded from the genotype HT 016 (30.20) followed by HT 017 (24.80), C 11 (23.42), HT 019 (21.77), C 21 (21.13), C 51 (19.13) and C 41 (18.20) showing high degree of heat tolerance during summer. These genotypes also produced considerable number of fruits/plant during winter. On the other hand though the number of fruits /plant during summer was very low in the genotypes TMS 013, TMS 013, VRT 001, VRT 002, VRT 003, TMS 005, TMS 003, TMS 015 and TMS 008, they produced larger number of fruits per plant during winter. Therefore, these genotypes could be treated as moderate to highly sensitive to high temperature. These results are also inconformity with the findings of Ahmad (2002).

\subsection{Total soluble solids (\%)}

Seasonal variations significantly influenced the total soluble solids (TSS) content of fruits in all genotypes. Higher TSS \% was recorded in winter compared to summer season. The results from winter season showed the variation from $4.37 \%$ in TMS 008 to $5.67 \%$ in TMS 017, while it was $3.39 \%$ in TMS 013 to $4.77 \%$ in BARI Tomato6 during summer (Table 2). Ahmad (2002) also found higher TSS (\%) during winter compared to summer.

\subsection{Fruit weight (g)}

Significant variations were exhibited in fruit weight in both the seasons (Table 3). In winter, larger fruits were produced by all the genotypes compared to summer. Went (1957) obtained three times larger fruits at $14{ }^{0} \mathrm{C}$ night temperature compared to $26{ }^{0} \mathrm{C}$ which are in consonance with the findings of the present investigation. The result obtained in the study showed that weight per fruit ranged from 11.46 to $98.50 \mathrm{~g}$ in summer, while it varied from 20.00 to $148.3 \mathrm{~g}$ in winter. The heaviest fruits were recorded from TMS 008 (98.50 g) followed by $71.58 \mathrm{~g}$ in VRT 001, $63.42 \mathrm{~g}$ in VRT 003. The genotype TMS 008 and HT 016 also produced the heaviest (148.3 g) and smallest (20.00 g) fruit, respectively during winter.

\subsection{Fruit yield / plant (g)}

As yield per plant was attributed with fruit weight, it varied significantly in both the seasons. In summer, the maximum (940.8 g) yield was recorded from the genotype $\mathrm{C} 11$ followed by C $51(738.5 \mathrm{~g}), \mathrm{C} 21(717 \mathrm{~g}), \mathrm{C} 41$ (692.7 g) and HT 019 (619.0 g) showing higher degree of heat tolerance among the genotypes (Table 3). On the other hand genotypes TMS 005, TMS 013, TMS 017 and TMS 011 produced lower fruit yield per plant indicating high degree of heat sensitivity (Table 5). The situation was reverse during winter. The maximum $(2670 \mathrm{~g})$ fruit yield per plant was recorded from the genotype VRT 003 followed by VRT 002 (2545 g) and TMS 008 (2513 g) (Table 3). These heat sensitive lines were found to produce $168.5 \mathrm{~g}, 129.4 \mathrm{~g}$ and $313.6 \mathrm{~g}$ fruits per plant, respectively during summer showing moderate degree of heat sensitivity (Table 3). Baki (1991) reported a yield of 410, 173 and 11 $\mathrm{g}$ under high temperature conditions $\left(39^{\circ} \mathrm{C}\right.$ day $/ 28^{\circ} \mathrm{C}$ night) depending on the level of heat tolerance of the genotypes.

\subsection{Fruit length (cm)}

Fruit length varied significantly irrespective of season but the increased length was observed in winter and it was much pronounced in heat sensitive genotypes compared to heat tolerant ones. In winter it varied from 3.24 to $6.09 \mathrm{~cm}$ and the longest fruit was found in the genotype VRT 001 followed by VRT $003(5.62 \mathrm{~cm})$ and TMS $008(5.47 \mathrm{~cm})$ while in summer the height fruit length $(5.03 \mathrm{~cm})$ was recorded from VRT 003 and the lowest $(1.47 \mathrm{~cm})$ was in HT 016 (Table 3). Sawhney and Polowick (1985) mentioned reduced fruit length under high temperature.

\subsection{Fruit diameter (cm)}

Trend in fruit diameter was almost similar as in fruit length. The widest $(6.80 \mathrm{~cm})$ and narrowest $(2.99 \mathrm{~cm})$ fruits were recorded from TMS 008 and HT 016, respectively in winter while during summer the widest $(4.14 \mathrm{~cm})$ fruit was found in VRT 001 followed by $4.08 \mathrm{~cm}$ in TMS 008 , and the narrowest $(1.33 \mathrm{~cm})$ fruit was recorded in HT 016 (Table 3). 
Table 3. Yield attributing performances of tomato genotypes during winter and summer seasons

\begin{tabular}{|c|c|c|c|c|c|c|c|c|}
\hline \multirow[t]{2}{*}{ Genotype } & \multicolumn{2}{|c|}{ Fruit weight $(\mathrm{g})$} & \multicolumn{2}{|c|}{ Fruit yield / plant (g) } & \multicolumn{2}{|c|}{ Fruit length $(\mathrm{cm})$} & \multicolumn{2}{|c|}{ Fruit Diameter $(\mathrm{cm})$} \\
\hline & Winter & Summer & Winter & Summer & Winter & Summer & Winter & Summer \\
\hline C 11 & $49.00 \mathrm{fg}$ & 40.08 e-h & $1929 \mathrm{c}-\mathrm{g}$ & $940.8 \mathrm{a}$ & $4.28 \mathrm{f}$ & $3.07 \mathrm{~g}-\mathrm{i}$ & $4.27 \mathrm{fg}$ & $2.63 \mathrm{ef}$ \\
\hline C 21 & $37.00 \mathrm{hi}$ & $33.58 \mathrm{~h}-\mathrm{k}$ & 1725 d-h & $717.0 \mathrm{~b}$ & $4.03 \mathrm{f}-\mathrm{h}$ & $2.86 \mathrm{i}$ & $3.95 \mathrm{f}-\mathrm{h}$ & $2.81 \mathrm{de}$ \\
\hline C 41 & $35.00 \mathrm{i}$ & $37.33 \mathrm{f}-\mathrm{j}$ & 1659 e-h & $692.7 \mathrm{~b}$ & $3.83 \mathrm{gh}$ & $2.05 \mathrm{j}$ & $3.86 \mathrm{gh}$ & $2.46 \mathrm{ef}$ \\
\hline C 51 & $59.50 \mathrm{ef}$ & $38.45 \mathrm{e}-\mathrm{i}$ & 2139 a-f & $738.5 b$ & $4.00 \mathrm{f}-\mathrm{h}$ & $1.97 \mathrm{jk}$ & $4.94 \mathrm{e}$ & $2.86 \mathrm{c}-\mathrm{e}$ \\
\hline C 61 & $34.67 \mathrm{e}$ & $28.34 \mathrm{~h}-1$ & $1224 \mathrm{~h}$ & $438.6 \mathrm{de}$ & $4.14 \mathrm{fg}$ & $2.92 \mathrm{hi}$ & $3.97 \mathrm{f}-\mathrm{h}$ & $2.60 \mathrm{ef}$ \\
\hline C 71 & 57.67 ef & $39.91 \mathrm{e}-\mathrm{h}$ & $1878 \mathrm{c}-\mathrm{g}$ & $436.0 \mathrm{de}$ & $5.23 \mathrm{~cd}$ & $4.65 \mathrm{ab}$ & $4.39 \mathrm{f}$ & $2.88 \mathrm{~b}-\mathrm{e}$ \\
\hline HT 016 & $20.00 \mathrm{j}$ & $11.46 \mathrm{~m}$ & $1627 \mathrm{f}-\mathrm{h}$ & $345.8 \mathrm{~d}-\mathrm{f}$ & $3.32 \mathrm{i}$ & $1.47 \mathrm{k}$ & $2.99 \mathrm{i}$ & $1.33 \mathrm{~g}$ \\
\hline HT 017 & $22.33 \mathrm{j}$ & $19.30 \mathrm{~lm}$ & 1985 b-g & $483.4 \mathrm{~cd}$ & $3.24 \mathrm{i}$ & $2.14 \mathrm{j}$ & $3.24 \mathrm{i}$ & $2.03 \mathrm{f}$ \\
\hline HТ 019 & $33.33 \mathrm{i}$ & $32.23 \mathrm{~h}-1$ & $1456 \mathrm{gh}$ & $619.0 \mathrm{bc}$ & $3.97 \mathrm{f}-\mathrm{h}$ & $2.91 \mathrm{hi}$ & $3.70 \mathrm{~h}$ & $2.46 \mathrm{ef}$ \\
\hline VRT 001 & $89.26 \mathrm{c}$ & $71.58 \mathrm{~b}$ & $2358 \mathrm{a}-\mathrm{c}$ & $109.4 \mathrm{gh}$ & $6.09 \mathrm{a}$ & $4.33 \mathrm{bc}$ & $5.42 \mathrm{~cd}$ & $4.14 \mathrm{a}$ \\
\hline VRT 002 & $88.67 \mathrm{~cd}$ & $48.28 \mathrm{~d}-\mathrm{g}$ & $2545 \mathrm{a}$ & $129.4 \mathrm{gh}$ & $5.40 \mathrm{bc}$ & $3.77 \mathrm{~d}-\mathrm{f}$ & $5.46 \mathrm{~cd}$ & $2.57 \mathrm{ef}$ \\
\hline VRT 003 & $87.33 \mathrm{~cd}$ & $63.42 \mathrm{bc}$ & $2670 \mathrm{a}$ & $168.5 \mathrm{f}-\mathrm{h}$ & $5.62 \mathrm{~b}$ & $5.03 \mathrm{a}$ & $5.14 \mathrm{de}$ & $2.78 \mathrm{de}$ \\
\hline TMS 003 & $78.67 \mathrm{~d}$ & $55.83 \mathrm{~cd}$ & 2265 a-c & $95.5 \mathrm{~h}$ & $4.76 \mathrm{e}$ & 3.47 e-h & $5.19 \mathrm{c}-\mathrm{e}$ & $3.55 \mathrm{ab}$ \\
\hline TMS 005 & $45.67 \mathrm{gh}$ & $24.75 \mathrm{j}-1$ & $1252 \mathrm{~h}$ & $42.2 \mathrm{~h}$ & $3.69 \mathrm{~h}$ & $2.23 \mathrm{j}$ & $4.33 \mathrm{f}$ & $2.58 \mathrm{ef}$ \\
\hline TMS 008 & $148.33 \mathrm{a}$ & $98.50 \mathrm{a}$ & $2513 \mathrm{ab}$ & $313.6 \mathrm{~d}-\mathrm{f}$ & $5.47 \mathrm{bc}$ & $4.22 \mathrm{~b}-\mathrm{d}$ & $6.80 \mathrm{a}$ & $4.08 \mathrm{a}$ \\
\hline TMS 011 & $99.33 \mathrm{~b}$ & $50.18 \mathrm{~d}-\mathrm{f}$ & 2183 a-e & $92.25 \mathrm{~h}$ & $4.98 \mathrm{de}$ & $3.30 \mathrm{f}-\mathrm{i}$ & $5.99 \mathrm{~b}$ & $3.50 \mathrm{abc}$ \\
\hline TMS 013 & $51.00 \mathrm{c}-\mathrm{g}$ & $21.71 \mathrm{k}-\mathrm{m}$ & $1241 \mathrm{~h}$ & $42.95 \mathrm{~h}$ & $3.69 \mathrm{~h}$ & $1.82 \mathrm{jk}$ & $4.34 \mathrm{f}$ & $2.34 \mathrm{ef}$ \\
\hline TMS 015 & $50.24 \mathrm{c}-\mathrm{g}$ & $35.50 \mathrm{~g}-\mathrm{j}$ & $1546 \mathrm{gh}$ & $37.3 \mathrm{~h}$ & $5.17 \mathrm{~cd}$ & $3.98 \mathrm{c}-\mathrm{e}$ & $4.25 \mathrm{fg}$ & $2.93 \mathrm{~b}-\mathrm{e}$ \\
\hline TMS 017 & $88.33 \mathrm{c}-\mathrm{d}$ & $25.33 \mathrm{i}-1$ & 2230 a-d & $49.2 \mathrm{~h}$ & $5.37 \mathrm{bc}$ & $2.78 \mathrm{i}$ & $5.18 \mathrm{c}-\mathrm{e}$ & $2.38 \mathrm{ef}$ \\
\hline BARI 6 & $92.67 \mathrm{bc}$ & $51.44 \mathrm{c}-\mathrm{e}$ & $2318 \mathrm{a}-\mathrm{c}$ & $282.3 \mathrm{e}-\mathrm{g}$ & $5.01 \mathrm{de}$ & $3.58 \mathrm{e}-\mathrm{g}$ & $5.58 \mathrm{c}$ & $3.36 \mathrm{~b}-\mathrm{d}$ \\
\hline $\mathrm{CV}(\%)$ & 8.81 & 16.93 & 4.56 & 29.64 & 4.17 & 9.93 & 5.00 & 13.3 \\
\hline Level of significance & $* *$ & $* *$ & $* *$ & $* *$ & $* *$ & $* *$ & $* *$ & $* *$ \\
\hline
\end{tabular}

Means in a column followed by the same letters are not significantly different at $1 \%$ level of probability by DMRT. 
Table 4. Performance of tomato genotypes during winter and summer seasons for primary branches per plant, plant height, seed /fruit and 1000 seed weight

\begin{tabular}{|c|c|c|c|c|c|c|c|c|}
\hline \multirow[t]{2}{*}{ Genotype } & \multicolumn{2}{|c|}{ primary branches/plant } & \multicolumn{2}{|c|}{ Plant height $(\mathrm{cm})$} & \multicolumn{2}{|c|}{ Seeds/fruit } & \multicolumn{2}{|c|}{1000 seed weight $(\mathrm{g})$} \\
\hline & Winter & Summer & Winter & Summer & Winter & Summer & Winter & Summer \\
\hline C 11 & $3.55 \mathrm{ab}$ & 4.60 a-e & 83.33 ef & $140.5 \mathrm{e}-\mathrm{g}$ & $46.90 \mathrm{~h}$ & $19.18 \mathrm{de}$ & $2.44 \mathrm{e}-\mathrm{h}$ & $2.28 \mathrm{de}$ \\
\hline C 21 & $3.67 \mathrm{a}$ & $4.77 \mathrm{a}-\mathrm{e}$ & $68.40 \mathrm{~g}$ & $127.9 \mathrm{fg}$ & $51.30 \mathrm{gh}$ & $24.74 \mathrm{bc}$ & $2.76 \mathrm{~cd}$ & $2.17 \mathrm{e}-\mathrm{g}$ \\
\hline C 41 & $3.42 \mathrm{a}-\mathrm{c}$ & $4.25 \mathrm{~b}-\mathrm{e}$ & 96.53 b-e & $151.6 \mathrm{c}-\mathrm{g}$ & $31.30 \mathrm{j}$ & 15.96 ef & $2.80 \mathrm{bc}$ & $2.68 \mathrm{~b}$ \\
\hline C 51 & $3.47 \mathrm{ab}$ & $4.27 \mathrm{~b}-\mathrm{e}$ & $81.53 \mathrm{f}$ & $151.8 \mathrm{c}-\mathrm{g}$ & $84.30 \mathrm{~d}$ & $22.67 \mathrm{~b}-\mathrm{d}$ & $3.21 \mathrm{a}$ & $3.02 \mathrm{a}$ \\
\hline C 61 & $3.53 \mathrm{ab}$ & 5.07 a-e & $90.67 \mathrm{~b}-\mathrm{f}$ & $170.1 \mathrm{~b}-\mathrm{e}$ & $40.10 \mathrm{i}$ & $11.05 \mathrm{gh}$ & $3.16 \mathrm{a}$ & $2.93 \mathrm{a}$ \\
\hline C 71 & $2.47 \mathrm{de}$ & $4.18 \mathrm{c}-\mathrm{e}$ & $87.20 \mathrm{c}-\mathrm{f}$ & $158.9 \mathrm{c}-\mathrm{f}$ & $60.60 \mathrm{f}$ & $7.17 \mathrm{~h}-\mathrm{j}$ & $2.43 \mathrm{e}-\mathrm{h}$ & $2.45 \mathrm{~cd}$ \\
\hline НТ 016 & 3.20 a-e & 4.88 a-e & $102.60 \mathrm{~b}$ & $203.5 \mathrm{ab}$ & $26.00 \mathrm{j}$ & $4.50 \mathrm{ij}$ & $2.40 \mathrm{f}-\mathrm{h}$ & $2.12 \mathrm{e}-\mathrm{g}$ \\
\hline HТ 017 & $3.40 \mathrm{a}-\mathrm{c}$ & 4.80 a-e & $101.67 \mathrm{~b}$ & $198.9 \mathrm{ab}$ & $31.20 \mathrm{j}$ & $21.56 \mathrm{~cd}$ & $2.82 \mathrm{bc}$ & $2.24 \mathrm{ef}$ \\
\hline НТ 019 & $3.53 \mathrm{ab}$ & $4.35 \mathrm{~b}-\mathrm{e}$ & $69.53 \mathrm{~g}$ & $145.0 \mathrm{~d}-\mathrm{g}$ & $63.20 \mathrm{f}$ & $14.27 \mathrm{fg}$ & $2.59 \mathrm{de}$ & $2.06 \mathrm{fg}$ \\
\hline VRT 001 & $3.27 \mathrm{a}-\mathrm{d}$ & $3.95 \mathrm{e}$ & $85.87 \mathrm{~d}-\mathrm{f}$ & $147.4 \mathrm{c}-\mathrm{g}$ & $71.20 \mathrm{e}$ & $11.67 \mathrm{f}-\mathrm{h}$ & $2.86 \mathrm{bc}$ & $2.05 \mathrm{~g}$ \\
\hline VRT 002 & $3.02 \mathrm{a}-\mathrm{e}$ & $4.80 \mathrm{a}-\mathrm{e}$ & $91.07 \mathrm{~b}-\mathrm{f}$ & $153.6 \mathrm{c}-\mathrm{g}$ & $80.13 \mathrm{~d}$ & $13.98 \mathrm{fg}$ & $2.60 \mathrm{de}$ & $2.60 \mathrm{bc}$ \\
\hline VRT 003 & $2.80 \mathrm{~b}-\mathrm{e}$ & 5.07 a-e & $96.33 \mathrm{~b}-\mathrm{e}$ & $181.6 \mathrm{bc}$ & $72.30 \mathrm{e}$ & $10.74 \mathrm{gh}$ & $2.57 \mathrm{ef}$ & $2.48 \mathrm{c}$ \\
\hline TMS 003 & $3.20 \mathrm{a}-\mathrm{e}$ & $4.82 \mathrm{a}-\mathrm{e}$ & $100.47 \mathrm{bc}$ & $181.2 \mathrm{bc}$ & $100.80 \mathrm{~b}$ & $26.97 \mathrm{~b}$ & $2.80 \mathrm{bc}$ & $1.60 \mathrm{~h}$ \\
\hline TMS 005 & $2.40 \mathrm{e}$ & $5.53 \mathrm{a}$ & $97.93 \mathrm{~b}-\mathrm{d}$ & $176.3 b-d$ & $107.70 \mathrm{a}$ & $14.90 \mathrm{e}-\mathrm{g}$ & $2.29 \mathrm{~h}$ & $2.30 \mathrm{de}$ \\
\hline TMS 008 & $2.80 \mathrm{~b}-\mathrm{e}$ & $5.28 \mathrm{a}-\mathrm{c}$ & $102.47 \mathrm{~b}$ & $176.7 \mathrm{~b}-\mathrm{d}$ & $106.30 \mathrm{a}$ & $49.39 \mathrm{a}$ & $2.30 \mathrm{~h}$ & $2.23 \mathrm{e}-\mathrm{g}$ \\
\hline TMS 011 & $3.33 \mathrm{a}-\mathrm{c}$ & $5.33 \mathrm{ab}$ & $149.8 \mathrm{a}$ & $218.3 \mathrm{a}$ & $53.30 \mathrm{~g}$ & $9.00 \mathrm{hi}$ & $2.50 \mathrm{e}-\mathrm{g}$ & 2.24 ef \\
\hline TMS 013 & $3.13 \mathrm{a}-\mathrm{e}$ & $5.20 \mathrm{a}-\mathrm{d}$ & $87.73 \mathrm{c}-\mathrm{f}$ & $172.2 \mathrm{~b}-\mathrm{e}$ & $90.20 \mathrm{c}$ & $11.30 \mathrm{f}-\mathrm{h}$ & $2.35 \mathrm{gh}$ & $2.23 \mathrm{ef}$ \\
\hline TMS 015 & $2.82 \mathrm{~b}-\mathrm{e}$ & $5.13 \mathrm{a}-\mathrm{d}$ & 92.44 b-f & $121.3 \mathrm{~g}$ & $28.50 \mathrm{j}$ & $5.66 \mathrm{ij}$ & $2.89 \mathrm{bc}$ & $2.28 \mathrm{de}$ \\
\hline TMS 017 & $3.27 \mathrm{a}-\mathrm{d}$ & 4.72 & 84.60 d-f & $145.6 \mathrm{~d}-\mathrm{g}$ & $64.80 \mathrm{f}$ & $4.02 \mathrm{j}$ & $2.96 \mathrm{~b}$ & $1.53 \mathrm{~h}$ \\
\hline BARI 6 & $2.63 \mathrm{c}-\mathrm{e}$ & $4.13 \mathrm{de}$ & $146.20 \mathrm{a}$ & $173.1 \mathrm{~b}-\mathrm{e}$ & $50.30 \mathrm{gh}$ & $8.39 \mathrm{~h}-\mathrm{j}$ & $3.28 \mathrm{a}$ & $2.67 \mathrm{~b}$ \\
\hline $\mathrm{CV}(\%)$ & 13.23 & 12.13 & 7.49 & 10.9 & 5.25 & 8.60 & 3.72 & 4.26 \\
\hline $\begin{array}{l}\text { Level of } \\
\text { significance }\end{array}$ & $* *$ & $* *$ & $* *$ & $* *$ & $* *$ & $* *$ & $* *$ & $* *$ \\
\hline
\end{tabular}

Means in a column followed by the same letters are not significantly different at $1 \%$ level of probability by DMRT. 
Table 5. Monthly mean temperature and relative humidity during the crop period

\begin{tabular}{|c|c|c|c|c|c|}
\hline \multirow[t]{2}{*}{ Year } & \multirow[t]{2}{*}{ Month } & \multicolumn{2}{|c|}{ Temperature $\left({ }^{0} \mathrm{C}\right)$} & \multicolumn{2}{|c|}{ Relative humidity (\%) } \\
\hline & & Maximum & Minimum & Average & Rainfall (mm) \\
\hline \multirow[t]{4}{*}{2006} & September & 32.2 & 25.6 & 75.7 & 572.4 \\
\hline & October & 32.7 & 24.3 & 68.4 & 33.6 \\
\hline & November & 29.9 & 19.3 & 65.35 & 0.0 \\
\hline & December & 27.40 & 14.3 & 55.55 & 0.0 \\
\hline \multirow[t]{11}{*}{2007} & January & 24.8 & 11.4 & 61.65 & 0.0 \\
\hline & February & 27.2 & 15.9 & 64.35 & 76.6 \\
\hline & March & 31.4 & 17.8 & 60.25 & 14.8 \\
\hline & May & 34.70 & 25.40 & 67.30 & 191.60 \\
\hline & June & 32.3 & 25.5 & 75.70 & 726.2 \\
\hline & July & 31.6 & 25.70 & 80.45 & 687.80 \\
\hline & August & 32.30 & 26.70 & 74.95 & 299.00 \\
\hline & September & 32.00 & 26.40 & 75.50 & 145.80 \\
\hline & October & 32.10 & 23.50 & 38.85 & 393.30 \\
\hline & November & 29.40 & 19.5 & 37.30 & 63.0 \\
\hline & December & 26.00 & 13.80 & 62.10 & 0.0 \\
\hline \multirow[t]{10}{*}{2008} & January & 24.60 & 13.40 & 64.75 & 91.40 \\
\hline & February & 25.9 & 14.50 & 61.10 & 67.80 \\
\hline & March & 31.10 & 20.70 & 65.20 & 20.00 \\
\hline & April & 34.00 & 23.90 & 60.55 & 19.80 \\
\hline & May & 33.80 & 24.30 & 64.30 & 312.40 \\
\hline & June & 31.4 & 25.8 & 77.00 & 391.40 \\
\hline & July & 31.01 & 26.1 & 79.8 & 440.20 \\
\hline & August & 31.28 & 25.88 & 82.18 & 887.15 \\
\hline & September & 32.32 & 25.8 & 77.90 & 871.95 \\
\hline & October & 31.45 & 23.42 & 79.36 & 850.6 \\
\hline
\end{tabular}

\subsection{Primary branches / plant}

Like other parameters, the genotypes differed significantly in both the season in respect of primary branches per plant (Table 4). All the genotypes produced higher number of primary branches in summer compared to those in winter. This might be due to taller plants at high temperature under polytunnel during summer. In summer primary branches per plant ranged from 3.95 in VRT 001 to 5.53 in TMS 005 while, in winter it ranged from 2.40 in TMS 005 to 3.67 in C 21.

\subsection{Plant height (cm)}

Wide variation among the genotypes was observed in respect of plant height in both the seasons, but increased plant height was found in summer (Table 4). The tallest plants $149.8 \mathrm{~cm}$ in winter and $218.3 \mathrm{~cm}$ in summer were recorded from the same genotype (TMS 008) but the shortest $(68.40 \mathrm{~cm})$ was recorded from C 21 in winter and $121.3 \mathrm{~cm}$ from TMS 015 in summer.

\subsection{Seeds per fruit}

Table 4 shows highly significant variations in number of seeds per fruit in both the seasons among the genotypes. Number of seeds per fruit was many folds higher during winter compared to that in summer in all the genotypes. This might be due to increased pollen viability during winter which facilitated the maximum pollen tube growth through the style into the ovary along with other favorable physiological factors for embryological process under optimum 
temperature (Table 5). In winter it ranged from 26.00 to 107.70 in the genotypes HT 016 and TMS 005, respectively while it varied from 4.02 to 49.39 in the genotypes TMS 017 and TMS 008, respectively. The results revealed from the present investigation are in agreement with the findings of Ahmad (2002).

\subsection{0 seed weight (g)}

All the genotypes varied significantly in respect of 1000 seed weight in both the seasons (Table 4) but it was higher during winter with few exceptions (C 71 and TMS 008) compared to that in summer. The ranges of 1000 seed weight were from 2.29 to $3.28 \mathrm{~g}$ during winter and it was 1.53 to $3.02 \mathrm{~g}$ during summer. Higher seed weight during winter might be due to increased synthesis of plant hormones along with other favourable factors for seed formation under optimum temperature.

\section{Conclusions}

From the above results it was revealed that wide ranges of variabilities were existed among the 20 genotypes in respect of tomato for yield and yield contributing characters. The genotypes also showed seasonal variations for all the parameters. In summer, the maximum (940.8 g) yield was recorded from the genotype $\mathrm{C} 11$ followed by C 51 (738.5 g), C 21 (717 g), C 41 (692.7 g) and HT 019 (619.0 g) showing higher degree of heat tolerance among the genotypes. On the other hand, the genotypes TMS 005, TMS 013, TMS 017 and TMS 011 produced lower fruit yield per plant indicating high degree of heat sensitivity. The situation was reverse during winter.

\section{References}

Ahmad, S. 2002. Genetics of fruit set and related traits in tomato under hot humid conditions. Ph D Thesis. Bangabandhu Sheikh Mujibur Rahman Agricultural University, Salna, Gazipur. 1-62 pp.
Anonymous. 1995. Agro-climatatrological data. Agromet Division, Bangladesh Meteorological Department, Joydebpur, Gazipur. 35-65 pp.

Baki, A. A. 1991. Tolerance of tomato cultivars and selected germplasm to heat stress. Journal of the American Society for Horticultural Science, USDA- ARS Vegetable. 48-55 pp.

BARI. 2011. Tomato. In: Mondal, M. R. I., M. S. Islam, M. A. J. Bhuyan, M. M. Rahman and M. H. H. Rahman (Eds.). Krishi Projukti Hatboi. Part-1, $5^{\text {th }}$ edn. Bangladesh Agricultural Research Institute. Gazipur, 1701. Bangladesh. 372$396 \mathrm{pp}$.

Bodo, R. T. 1991. Comparison of different pollen viability assays to evaluate pollen fertility of potato diploids. Euphytica. 56: 143-148.

Charles, W. B. and R. E. Harris. 1972. Tomato fruit set at high and low temperatures. Canadian Journal of Plant Science, 52: 497-506.

Hanna, H. Y. and Hernandez, T. P. 1979. Heat tolerance in tomato. Horticulture Science, 14 (2): 121.

Kuo, C. G., B. W. Chen, M. H. Chou, C. C. Tsai and J. S. Tsay. 1979. Tomato fruit set at high temperature. In: Cowel R. (ed.) Proc. $1^{\text {st }}$ Intl. Symp. Tropical Tomato. Asian Vegetable Research and Development Center, Shanhua, Taiwan. 94-108 pp.

Sawhney, V. K. and P. L. Polowick. 1985. Fruit development in tomato: The role of temperature. Canadian Journal of Botany, 63: 1031-1034.

Went, F. W. 1957. The experimental control of plant growth. Chronica Botanica Co., Waltham, Mass. 99-114 pp. 